\title{
INNOVATIONS IN THE MATHEMATICS TEACHING IN SECONDARY SCHOOL
}

\author{
Djoko G. Marković \\ University of Montenegro, Faculty of Philosophy, \\ 81460 Nikšić, Danila Bojovic bb, Montenegro,
}

djokogm@uninp.edu.rs

DOI:10.5901/mjss.2014.v5n19p619

\begin{abstract}
The subjects of research are concrete possibilities of use philosophical-historical-developed and methodological-innovative elements in the mathematics teaching in elementary and secondary school in breaking of formalism and activating e.g. dynamism of teaching. The aim is that the pupils think over with originality and creation thinking. It develops usage of indepedent thinking, critical estimation and reasonable generalization.
\end{abstract}

Keywords: Innovations, philosophical-historical-developed elements in the mathematics teaching, methodologicalinnovations, breaking of formalism, dynamism of teaching mathematics.

\section{Introduction}

Properties of important points of a triangle (orthocenter, center of gravity, centers of a circumscribed circle and centers of an inscribed circle) as well as methods of their construction have been known since ancient times. However, it is less known about the existence, properties and structures of some unusual points of a triangle, such as J. Steiner's (1796 1863) point (which has the property that the sum of the distance from it to the apex of a triangle is minimal), or H. Brocard's (1845 - 1922) point (with the property $\measuredangle \Omega A B=\measuredangle \Omega B C=\Varangle \Omega C A$ where $\Omega$ is the inner point of triangle $A B C$ ), or of some other less important points. [1]

On this occasion we will not deal with the well-known properties and structures of J. Steiner's point, nor with the properties and structures of Brocard's point of a triangle, but with properties and constructions of a less distinctive and less familiar point of a triangle for which the following theorem is true.

\section{Properties of an Unusual Point of a Triangle}

Theorem 1. If three congruent circumferences intersecting in point $S_{1}$ and each touching two sides of $\triangle A B C$ are inscribed in the triangle and if points $O$ and $S$ are the centers of the circumscribed and inscribed circles of $\triangle A B C$, then points $O, S$ and $S_{1}$ are collinear. 


\section{Proof:}

Let us designate with points $A_{1}, B_{1}$ and $C_{1}$ centers of the given congruent circumferences. It is easy to notice that they belong to the bisectors of the interior angles in $\triangle A B C$ (see figure 1). $A_{1} L=B_{1} G \Rightarrow \overline{A_{1} B_{1}} \| \overline{A B}$. In the similar way, we conclude that $\overline{A_{1} C_{1}} \| \overline{A C}$ and $\overline{B_{1} C_{1}} \| \overline{B C}$, from which follows that $\triangle A B C$ and $\triangle A_{1} B_{1} C_{1}$ have common bisectors of the interior angles, that is they have one common center $S$ of the inscribed circles. It is easy to note that a homothety $\mathrm{H}$ with center $S$ and coefficient $k$ maps $\triangle A_{1} B_{1} C_{1}$ into $\triangle A B C$, i.e. $\mathrm{H}$ ${ }_{s}^{k}\left(\triangle A_{1} B_{1} C_{1}\right)=\triangle A B C$, where $k=\frac{A B}{A_{1} B_{1}}$. However, since we have that, according to the given problem, $S_{1} A_{1}=S_{1} B_{1}=S_{1} C_{1}$, point $S_{1}$ is the center of the circumscribed circle around $\triangle A_{1} B_{1} C_{1}$. Since $\triangle A B C$ and $\triangle A_{1} B_{1} C_{1}$ are homothetic, the circles circumscribed around them are also homothetic, therefore we have that $\mathrm{H}$ ${ }_{S}^{k}\left(S_{1}\right)=O$, i.e. points $O, S_{1}$ and $S$ are collinear, which was to be proven.

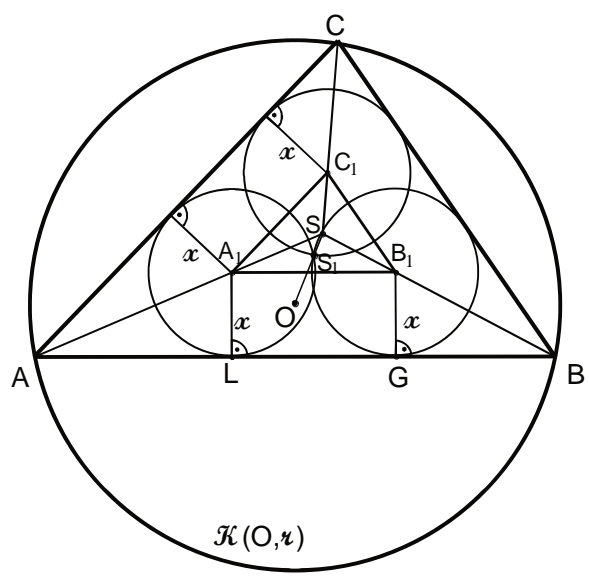

Figure 1.

Property of colinearity of points $O, S_{1}$ and $S$ enables us to construct point $S_{1}$, that is to solve the following problem.

\section{Constructions of an unusual point of a triangle}

2. Inscribe (construct) in the given triangle $A B C$ three congruent circumferences intersecting in point $S_{1}$, where each circumference touches two sides of the triangle. 
Analysis: According to the previous theorem, we have that a homothety $\mathrm{H}$ with center $S$ and coefficient $k$ maps $\triangle A_{1} B_{1} C_{1} \quad$ into $\triangle A B C, \quad$ i.e. $\quad H_{S}^{k}\left(\triangle A_{1} B_{1} C_{1}\right)=\triangle A B C, \quad$ where $k=\frac{A B}{A_{1} B_{1}}=\frac{B C}{B_{1} C_{1}}=\frac{C A}{C_{1} A_{1}}=\frac{S A}{S A_{1}}=\frac{S O}{S S_{1}}$ (see figure 1).

In order to construct the requested point $S_{1}$ of the given triangle it is necessary and sufficient to find the homothety (similarity) coeficient.

For that purpose, we construct a figure similar (homothetic) to the one in picture 1. That figure given in picture 2 consists of a pair of homothetic triangles $\triangle A_{0} B_{0} C_{0}$ and $\triangle A_{2} B_{2} C_{2}$ which due to similarity to the figure in picture 1, i.e. similarity to a corresponding pair of homothetic triangles $\triangle A B C$ and $\triangle A_{1} B_{1} C_{1}$ have the same homothety coefficient $k$.

We obtain this figure by constructing in the following way:

First let us construct any $\triangle A_{0} B_{0} C_{0}$ which is similar to a pair of homothetic triangles $\triangle A B C$ and $\triangle A_{1} B_{1} C_{1}$, and which will concurrently be homothetic with triangle $A_{2} B_{2} C_{2}$, where the center of that homothety is point $S_{0}$, and coefficient $k$, i.e. $\mathrm{H}_{S_{0}}^{k}\left(\Delta A_{0} B_{0} C_{0}\right)=\Delta A_{2} B_{2} C_{2}$.

Let us construct centers of inscribed and circumscribed circles in and around $\triangle A_{0} B_{0} C_{0}$, that is let us construct bisectors of its interior angles in whose intersection is found point $S_{0}$ - the center of the circle inscribed in $\Delta A_{0} B_{0} C_{0}$, and let us construct bisectors of its sides in whose intersection is found point $S_{2}$ - the center of its circumscribed circle (see figure 2). Let us construct three congruent circumferences which have one common point $S_{2}$ (the center of the circumscribed circle around $\Delta A_{0} B_{0} C_{0}$ ), with centers $A_{0}, B_{0}$ and $C_{0}$ and with equal radiuses $A_{0} S_{2}=B_{0} S_{2}=C_{0} S_{2}=y$. It is easy to construct common tangents $\left(A_{2} B_{2}\right),\left(A_{2} C_{2}\right)$ and $\left(B_{2} C_{2}\right)$ on pairs of those circumferences that generate triangle $A_{2} B_{2} C_{2}$ homothetic with $\triangle A_{0} B_{0} C_{0}$.

Since the homothetic figure in picture 2 is constructed in the way that it is similar to the homothetic figure in picture 1 , it means that the homothety coefficent is.

$$
k=\frac{A B}{A_{1} B_{1}}=\frac{B C}{B_{1} C_{1}}=\frac{C A}{C_{1} A_{1}}=\frac{S A}{S A_{1}}=\frac{S O}{S S_{1}}=\frac{A_{2} B_{2}}{A_{0} B_{0}}=\frac{B_{2} C_{2}}{B_{0} C_{0}}=\frac{C_{2} A_{2}}{C_{0} A_{0}}=\frac{S_{0} A_{2}}{S_{0} A_{0}}=\frac{S_{0} O_{0}}{S_{0} S_{2}}
$$




\section{Construction (first way):}

Through analysis we found that a pair of homothetic triangles $\left(\Delta A_{0} B_{0} C_{0}\right.$ and $\Delta A_{2} B_{2} C_{2}$ ) of the figure in picture 2 has the homothety coefficient that equals the one of a pair of corresponding homothetic triangles ( $\triangle A B C$ and $\triangle A_{1} B_{1} C_{1}$ ) of the figure in picture 1, i.e.

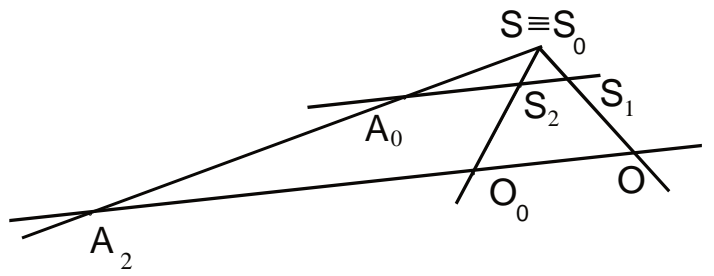

Figure 3.

$k=\frac{S_{0} A_{2}}{S_{0} A_{0}}=\frac{S_{0} O_{0}}{S_{0} S_{2}}=\frac{S_{0} O}{S S_{1}}$, therefore we can easily construct the requested segment $S_{0} S_{1}$.

Let us observe a part of the construction in figure 2, precisely let us construct a pair of corresponding homothetic triangles separately $\left(\Delta S_{0} A_{2} O_{0}\right.$ and $\left.\Delta S_{0} A_{0} S_{2}\right)$ (see figure 3).

Triangle $A B C$ is given and we can easily construct points $S$ (the intersection of bisectors of its interior angles) and $O$ (the intersection of bisectors of its sides) (see figure 4). Homothety $\mathrm{H}$ with center $S$ and coefficient $k$ maps $\Delta A_{1} B_{1} C_{1}$ into $\triangle A B C$, i.e. $H_{S}^{k}\left(\triangle A_{1} B_{1} C_{1}\right)=\triangle A B C$, where $k=\frac{A B}{A_{1} B_{1}}=\frac{B C}{B_{1} C_{1}}=\frac{C A}{C_{1} A_{1}}=\frac{S A}{S A_{1}}=\frac{S O}{S S_{1}}$.

Therefore, in figure 4, point $S_{1}$ is to be constructed, that is the segment $S S_{1}=S_{0} S_{1}$. As we constructed a segment $S O$ and since $k=\frac{S O}{S S_{1}}$, we can circumscribe in figure 3 a circle $\mathrm{K}\left(S_{0}, S_{0} O\right)$ with a center in the homothety center $S \equiv S_{0}$ and a radius $S_{0} O=S O$. With this homothety, points $A_{0}, S_{2}, S_{1}$ match points $A_{2}, O_{0}, O$, i.e. $\mathrm{H}_{S}^{k}\left(\Delta A_{2} \mathrm{O}_{0} O\right)=\Delta A_{0} S_{2} S_{1}$. Point $S_{1}$ is obtained as intersection of straight lines $\left(S_{0} O\right)$ and $\left(A_{0} S_{2}\right)$, i.e. $S_{1}=\left(S_{0} O\right) \cap\left(A_{0} S_{2}\right)$. Thus we constructed segment $S_{0} S_{1} \cong S S_{1}$ and on segment $S O$ we easily find the requested point $S_{1}$ of triangle $A B C$. In the similar way we can construct vertexes of $\triangle A_{1} B_{1} C_{1}$, and thus the three congruent circumferences in $\triangle \mathrm{ABC}$ that have the common intersection in point $S_{1}$ (figure 4). 


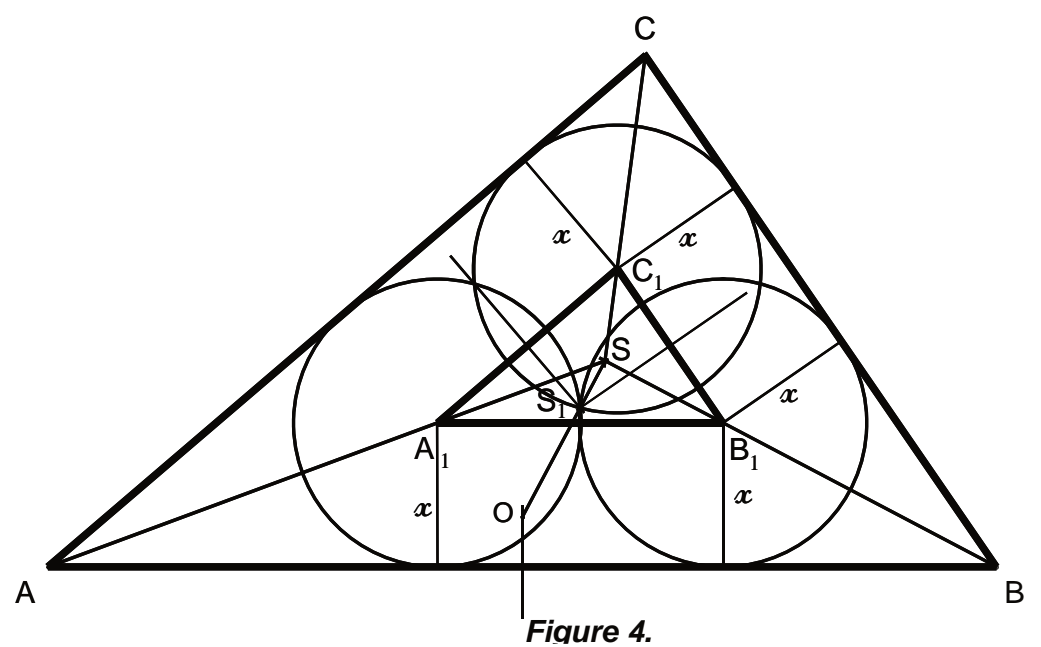

Construction of the requested point could have been done in the following way as well:

Let us construct again a pair of homothetic triangles $\left(\triangle A_{0} B_{0} C_{0}\right.$ and $\Delta A_{2} B_{2} C_{2}$ ) of the figure as in picture 2, and then let us construct $\triangle A B C$ in homothety with $\triangle A_{2} B_{2} C_{2}$. It is clear that in this case their homologous (corresponding) sides are parallel. Center $V$ of a new homothety is found in intersection of pencils of straight lines $\left(A A_{2}\right) \cap\left(B B_{2}\right) \cap\left(C C_{2}\right) \cap\left(S S_{0}\right)=V$, where the coefficient of this homothety (similarity) is $k_{1}=\frac{V A}{V A_{2}}=\frac{V B}{V B_{2}}=\frac{V C}{V C_{2}}=\frac{V S}{V S_{0}}$, then we have $H_{V}^{k_{1}}(\triangle A B C)=\Delta A_{2} B_{2} C_{2}$ and $H_{V}^{k_{1}}\left(S_{1}\right)=S_{2}$. Therefore, point $S_{1}$ is found in intersection of straight lines $\left(V S_{2}\right)$ and $(S O)$, i.e. $\left(V S_{2}\right) \cap(S O)=S_{1}$, and the construction of point $S_{1}$ is thus completed (see figure 5).

However, we have that $\mathrm{H}_{V}^{k_{1}}\left(\triangle A_{1} B_{1} C_{1}\right)=\Delta A_{0} B_{0} C_{0}$, and:

$\left(V A_{0}\right) \cap(S A)=A_{1},\left(V B_{0}\right) \cap(S B)=B_{1},\left(V C_{0}\right) \cap(S C)=C_{1}$, therefore we can construct point $S_{1}$ in a third way as a center of a circle circumscribed around $\Delta A_{1} B_{1} C_{1}$. 


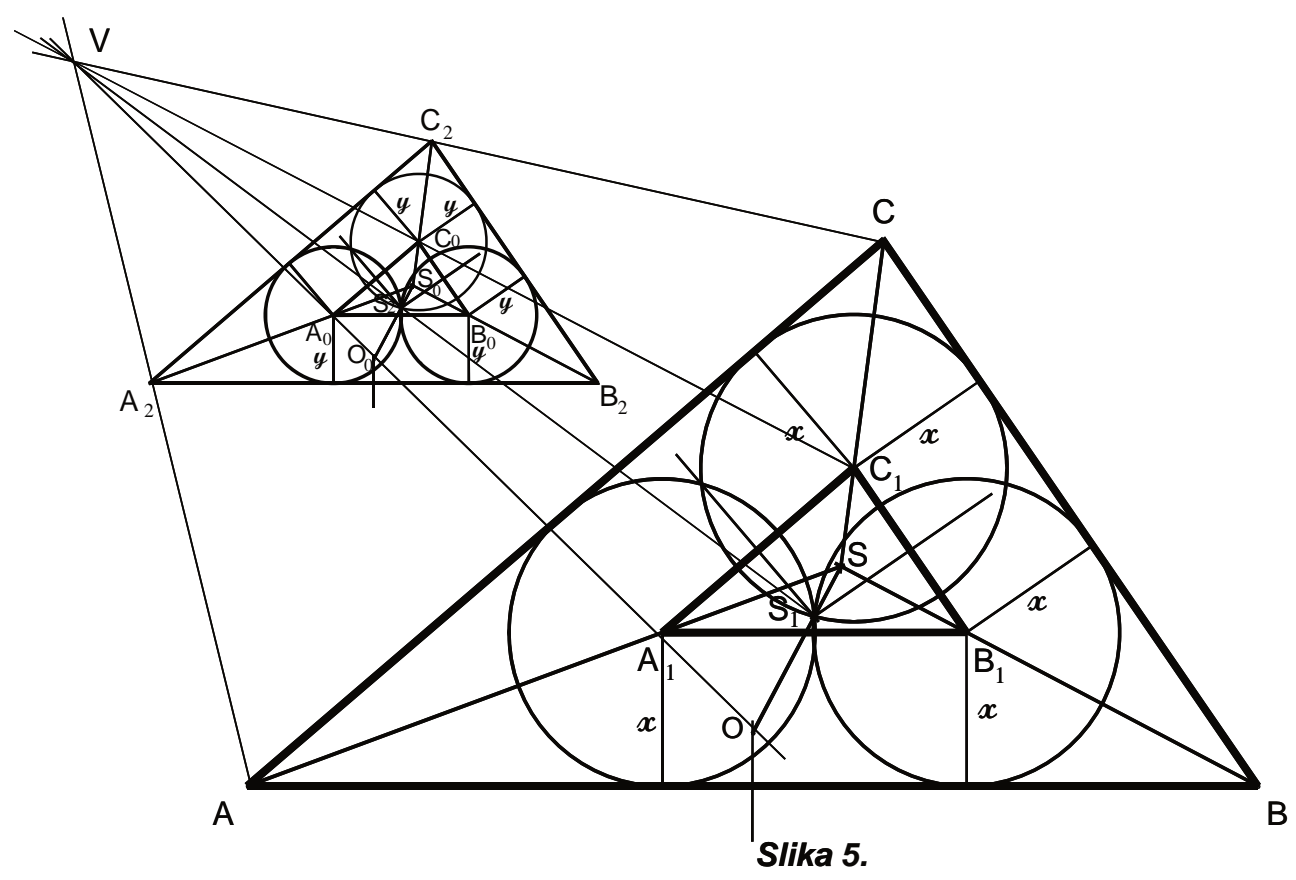

Proof:

Proof ensues from analysis and construction.

\section{Discussion:}

Since point $S_{1}$ as the center of the circumscribed circle around $\Delta A_{1} B_{1} C_{1}$ is single (only one), the problem has only one solution.

Such innovations presented in classes of teaching in high school result in students' increased interest in independent research or creative work and serve for eradicating formalism and activating or dynamazing the teaching process with talented students. 


\section{REFERENCES}

M. Bozic, (2002), Survey of the history and philosophy of mathematics, Institute for Textbooks and Teaching Aids, Belgrade.

G. Polya, (1966), How do / solve mathematical problems, school books, Zagreb, Croatia.

Marković, Đoko G. (2006). Geometrical polymorphism (Geometrijski polimorfiza.) (Serbian), Nast. Mat. 51, No. 3-4, 11-24.

Đ. G. Markovic, (2008), New views on methods of teaching mathematics, M3 Macarius, Podgorica. 